\title{
COMMENT
}

\section{Apoptosis inhibitor of macrophage and diabetic kidney disease}

\author{
Timothy M. E. Davis ${ }^{1}$, Kirsten E. Peters $\mathbb{D}^{1,2}$ and Richard Lipscombe ${ }^{2}$ \\ Cellular \& Molecular Immunology (2019) 16:521; https://doi.org/10.1038/s41423-018-0088-x
}

\section{Dear Editor,}

Miyazaki et al. recently reviewed the role of the apoptosis inhibitor of macrophage (AIM) protein, also known as CD5 antigen-like $(\mathrm{CD} 5 \mathrm{~L})$, as an apoptosis inhibitor that supports the survival of macrophages and protects against disease when it is in its free 'attacker' form after release from its circulating 'carrier' IgM. ${ }^{1}$ In particular, during acute kidney injury (AKI), evidence suggests that AIM is released systemically from the lgM pentamer, resulting in increased urinary excretion of free AIM, which exerts its local scavenging activity. ${ }^{2}$ We recently developed a protein biomarker test (PromarkerD) in which human plasma concentrations of AIM, apolipoprotein A-IV (ApoA4) and insulin-like growth factor binding protein 3 (IBP3) are measured using a mass spectrometry (MS) method. ${ }^{3}$ The method includes an immunodepletion step that removes lgM-bound AIM and measures only free AIM. As expected, ${ }^{1}$ we found that assayed concentrations of bound plus free AIM in whole plasma were 10- to 20-fold increased compared with those after immunodepletion and that free AIM concentrations were highest in young females.

In a cross-sectional analysis of a sample of 963 communitybased patients with type 2 diabetes, ${ }^{4}$ relative free AIM concentrations (derived from peak area ratios from the MS assay) were $25 \%$ increased in individuals with established renal disease (Kidney Disease: Improving Global Outcomes Chronic Kidney Disease Stage $\geq 1)^{5}$ versus those without (geometric mean (SD range) 1.90 (0.87-4.12) versus 1.51 (0.70-3.29)) after adjustment for age, serum HDL-cholesterol and plasma concentrations of the other two biomarkers, APOA4 and IBP3 $(P<0.001)$. This diagnostic finding is consistent with the observation that a substantial amount of free AIM can be found in sera from humans with AKI via immunoblotting. ${ }^{1}$ However, the baseline free AIM in serum was also a significant independent but inverse predictor of a subsequent rapid declining eGFR in a subgroup of patients, $\sim 50 \%$ of whom had an eGFR $>60 \mathrm{~mL} / \mathrm{min} / 1.73 \mathrm{~m}^{2}$ at baseline, as identified by trajectory analysis. ${ }^{4}$

The fact that lower baseline circulating levels of AIM were observed in our patients whose kidney function then declined rapidly may appear paradoxical. However, as we suggested in our earlier report, ${ }^{4}$ it is possible that some patients with type 2 diabetes have a defect in AIM gene expression that predisposes them to the future adverse effects of diabetes on the kidney by consequently reducing plasma concentrations of AIM (total and free). Other possible explanations include altered lgM binding that restricts release of free AIM and thus attenuates its beneficial effects in response to ongoing renal injury, accelerated renal clearance of free AIM (which could reflect a period of hyperfiltration, an adverse prognostic indicator in its own right, ${ }^{6}$ ) accelerated
AIM metabolism or alternative (non-lgM) binding of circulating free AIM that is not modulated by renal disease.

In the PromarkerD prognostic test, the risk of future rapidly declining function is quantified by plasma concentrations of AIM (inversely), ApoA4 (positively) and IBP3 (inversely) in association with increasing age, reduced serum $\mathrm{HDL}$-cholesterol levels and a reduced estimated glomerular filtration rate. The test has been validated ${ }^{7}$ and may serve as a useful addition to the tools available to health care professionals managing patients with type 2 diabetes. In addition, it has been postulated that substances that promote AIM release from its lgM binding sites could prove useful therapeutically, ${ }^{1}$ and the PromarkerD test could identify those who could benefit most from this intervention.

\section{Compliance with ethical standards}

\section{ADDITIONAL INFORMATION}

Conflict of interest: Proteomics International and the University of Western Australia are owners of patent PCT/AU2011/001212 that relates to biomarkers described in this letter. T.M.E.D. is employed by the University of Western Australia, K.E.P. by Proteomics International, the University of Western Australia and R.J.L. by Proteomics International.

Publisher's note: Springer Nature remains neutral with regard to jurisdictional claims in published maps and institutional affiliations.

\section{REFERENCES}

1. Miyazaki T., Yamazaki T., Sugisawa R., Gershwin M. E., Arai S. AIM associated with the IgM pentamer: attackers on stand-by at aircraft carrier. Cell. Mol. Immunol. https://doi.org/10.1038/cmi.2017.141. in press.

2. Arai, S. et al. Apoptosis inhibitor of macrophage protein enhances intraluminal debris clearance and ameliorates acute kidney injury in mice. Nat. Med. 22, 183-193 (2016).

3. Bringans, S. D. et al. Comprehensive mass spectrometry based biomarker discovery and validation platform as applied to diabetic kidney disease. EuPA Open Proteom. 14, 1-10 (2017).

4. Peters, K. E. et al. Identification of novel circulating biomarkers predicting rapid decline in renal function in type 2 diabetes: The Fremantle Diabetes Study Phase II. Diabetes Care. 40, 1548-1555 (2017).

5. Kidney Disease: Improving Global Outcomes Chronic Kidney Disease Guideline Development Work Group M. KDIGO 2012 clinical practice guideline for the evaluation and management of chronic kidney disease. Kidney Int. Suppl. 3, 1-150 (2013).

6. Davis, T. M., Chubb, S. A. \& Davis, W. A. The relationship between estimated glomerular filtration rate trajectory and all-cause mortality in type 2 diabetes: The Fremantle Diabetes Study. Eur. J. Endocrinol. 175, 273-285 (2016).

7. Peters, K. E. et al. Novel circulating biomarkers predict rapidly declining renal function in type 2 diabetes: The Fremantle Diabetes Study. Diabetes; 66 (Suppl. 1A): Abstract 20-LB.

\footnotetext{
${ }^{1}$ Faculty of Health and Medical Sciences, Medical School, University of Western Australia, Fremantle, Western Australia, Australia and ${ }^{2}$ Proteomics International, Perth, Western Australia, Australia

Correspondence: Timothy M. E. Davis (tim.davis@uwa.edu.au)
}

Received: 10 June 2018 Accepted: 12 June 2018

Published online: 12 July 2018 